\title{
Efficacy and safety of knee flexion enhanced standardized home exercise program comparing supervised physiotherapy to improve mobility and quality of life after Total knee arthroplasty
}

\section{Tianyang $\mathrm{Xu}$}

Shanghai Tenth People's Hospital

Lin Fan

Shanghai Tenth People's Hospital

Dong Yang

Shanghai Tenth People's Hospital

Kaiyuan Liu

Shanghai Tenth People's Hospital

Qiuming Gao

Shanghai Tenth People's Hospital

Guodong Li (D litrue2004@163.com)

Shanghai Tenth People's Hospital

\section{Research article}

Keywords: standardized home exercise program, supervised physiotherapy, TKA, rehabilitation

Posted Date: July 1st, 2020

DOI: https://doi.org/10.21203/rs.3.rs-32993/v1

License: (c) (i) This work is licensed under a Creative Commons Attribution 4.0 International License.

Read Full License 


\section{Abstract}

Background: In the present study, we introduced a knee flexion enhanced standardized home exercise program assisted by a low stool and evaluate efficiency of this method in the management of rehabilitation after TKA.

Methods: Patients were recruited from July 2014 and December 2015, and randomized into two groups, a knee flexion enhanced standardized home exercise program group (group KFEH) and commonly-used supervised physiotherapy group (group SPT). Outcomes were evaluated by observing the joint function with the Knee Society score (KSS), Visual Analogue Scale (VAS), the Western Ontario and McMaster Universities Osteoarthritis Index (WOMAC) score, and ROM assessment at selected time points (preoperation and 1 week, 1 month, 3 month, 6 month and 1 year following post-operation).

Results: At last, we observed pain and functional improvements in both groups. Noninferiority was shown 12 months postoperatively for clinical outcomes except group KFEH had better ROM range at 1 month $(P<0.01)$. The absolute value of WOMAC and KSS were slight better in group KFEH without significant difference, and there was no difference about VAS between two groups as well as complication incidence rate. Additionally, home program would save patients' times and decrease economic burden. Patents would save 14 times postoperative rehabilitation in outhospital service, and cut down at least 782 RMB in total.

Conclusion: Taking the rehabilitation and economical efficiency, we recommend knee flexion enhanced home exercise program for TKA rehabilitation management.

\section{Background}

Osteoarthritis (OA) of the knee joint, a complex peripheral joint disorder, is one of the most prevalent degenerative chronic diseases, which dramatically affect daily activity and quality of life in elderly patients [1, 2]. Among all of the surgical and chemical therapeutic resolutions, total knee arthroplasty (TKA) is a highly successful and a world-widely-accepted surgical treatment for OA [3-7]. The surgical aspect of TKA, improving the anatomy of the knee joint, is the first part of the total process. Functional rehabilitation, an important measurement to evaluate a successful TKA surgery, often takes few months, if not, years. Physical therapy plays an integral role in promoting functional rehabilitation following TKA, which could significant improvements were observed in function, quadriceps strength, and length of stay [8-10]. After the surgical procedure, early and sufficient rehabilitation programs, including physical therapy, will be greatly helpful to restore the function and range of motion (ROM) [10]. These rehabilitation programs or physical therapy could and should be continued after hospital discharge [11].

The physical therapist-supervised program is a commonly used standard of functional rehabilitation for patients underwent TKA. Typically, this physical therapy program supervised by a physical therapist during the hospital stay and in an outpatient facility for about 10-20 weeks [10]. Obviously, this standard program needs assistance of licensed therapists and professional equipments, and should be performed 
in the hospital or an outpatient facility. Therefore, these post-hospitalization programs require more outpatient facility visits (2-3 times/week) and related costs [12]. Time and economic burdens have limited the use of these programs. Numerous efforts have been focused on studies about costeffectiveness analysis and controlling costs of therapist-guided rehabilitation programs. There has been also an urgent pursuit for low-cost and practicable alternatives, such as self-performed home programs [13-16]. Comparing to physical therapist-supervised programs, these self-performed home ones are easier to be accepted by more and more patients without temporal and spacious limitations as well as any further cost. Home exercise usually includes telecare and standard home program. One of the disadvantages of standard home program is that these home programs usually do not have standard protocols, which lead to huge variations in its rehabilitation-promoting effects. Additionally, it can't be well guaranteed by the patients themselves completing the home exercise program. According to our experience, some patients doing home exercise usually faced poor knee flexion, which influence patients' body function and satisfaction towards surgery. Thanks for technology success, home tele-rehabilitation guidance of TKA patients has developed from standardized home exercise recent years. But due to economic condition and medical input of local district, telecare is not used widespread. Therefore, a current challenge in the field is to introduce standardized self-performed home programs could reduce knee stiffness especially enhance knee flexion function, and the patients should be well trained before hospital discharge.

In the present prospective randomized positive-controlled clinical trial, we first introduced the knee flexion enhanced assisted standardized home exercise program, and the flexion practice is assisted by a low stool (Appendix I). Further observed its overall rehabilitation-promoting effect by using standardized supervised physiotherapy (SPT) as a positive control in knee joint OA patients underwent TKA. We hypothesized that the postoperative rehabilitation of group KFEH is as efficient as that of SPT group.

\section{Methods}

This prospective randomized positive-controlled clinical trial was approved by the Institutional Medical Ethics Committee of our hospital, and was conducted in accordance with the approved guidelines. The trial is registered in the Chinese Clinical Trial Registry (http://www.chictr.org.cn/, ChiCTR-IOR-17011264). Informed consent was obtained from all of the subjects. TKA was performed through midline vertical incision and medial parapatellar approach by 2 chief surgeons who were highly experienced.

Posteriorcruciate stabilizing prostheses were implanted in all patients, without the patella resurfaced in the whole patients, and local infiltration analgesia around surgery fields were performed as previously reported $[13,14]$. Incision closure and wound care were performed in a standard manner in all of the patients. After surgery, all patients were given $50 \mathrm{mg}$ flurbiprofen axetil injection bid for a week to relieve pain, 10-mg rivaroxaban for 2 weeks to prevent deep vein thrombosis, and 1.5-g cefuroxime twice for 3 days to prevent infection. Patients were given pethidine $50 \mathrm{mg}$ for intolerable pain. Each patient was given 7 days inhospital assisted physical therapy since postoperative day 1 . To compensate for dropouts and deviation from normality, one hundred and sixty osteoarthritis patients scheduled for TKA between July 2014 to December 2015 were enrolled in the study. Patients were excluded if they were: younger than 
40 years or older than 80 years, revision surgery, lower limb ischemia, acute trauma or fracture, systemic or neuromuscular diseases, and mental disorders. Forty-one patients were excluded due to ineligibility. Finally, 119 of the enrolled patients were randomized to the group SPT $(n=60)$, or group KFEH $(n=59)$ using a computer-generated randomized number table (Fig. 1). Patients in the group SPT were instructed in a formal physical therapy program, and those from the group KFEH receiving home rehabilitation taught before TKA surgery as described in Appendix I. During their period of hospitalization, all of the enrolled patients underwent the same functional exercise-based rehabilitation program with the aim of improving knee range of motion, increasing knee and hip muscle strength, maintaining the length and elasticity of thigh tissues, preventing thrombosis, and acquiring the most important functional strategies. After discharge from the hospital, Patients in therapist-supervised physiotherapy group participated in a total of 24 sessions of a physiotherapy and rehabilitation program: 2 days/week for the first 7 weeks,followed by 1 day/month for the rest 10 months of the year. This program consists of knee joint range of motion (ROM) exercises and strengthening exercises (includes quadriceps setting exercise, straight leg raising exercise, stationary biking as previously described[17], training strength and length were individually designed) for the knee joint following $20 \mathrm{~min}$ of application of moist heat, and $20 \mathrm{~min}$ of transcutaneous electrical nerve stimulation (TENS) application. Knee flexion enhanced standardized home exercises included arrangement of knee joint motion, restoration of knee and hip muscle power. This program needs at least 5 days a week, 20 min/day, for 7 weeks, and 2-3 days a week for last 10 months. Home program consisted of quadriceps femoris (QF) sets, hamstring sets, ankle pumps, terminal knee extension with weight, straight leg raising with weight in supine, side-lying, cycling, and prone, hip and knee flexion-extension with weight in supine, knee flexion-extension with weight in prone, and in sitting, static stretching exercises for hamstrings and gastrosoleous muscles [13,18], as well as a low stool assisted knee joint bending exercise (Appendix I).

After enrollment, demographic and knee joint function-related information were collected by an orthopedist. For knee joint function measurements, patients from both groups underwent evaluations including the Knee Society score (KSS), the Western Ontario and McMaster Universities Osteoarthritis Index (WOMAC) score, ROM assessment at selected time points (pre-operation and 1 week, 1 month, 3 month, 6 month and 1 year following post-operation) as previously reported [11]. Visual Analogue Scale (VAS), one of the largely used pain scales, was used to evaluate painful behaviors at the statement of movement at different time points [19]. Patients from both groups were instructed to all of the measuring scales after enrollment. To reduce the subjective bias, all of the questionnaires were performed by different orthopedists.

\section{Statistics Analysis}

All of the data in the present study were recorded as mean \pm SD, and analyzed with SPSS 20.0. KSS, WOMAC, ROM and VAS were compared with unpaired student's t test. KSS, WOMAC, ROM and VAS at different time points between two groups following surgery were compared with two-way ANOVA, followed by Bonferroni's post-test. All data with $\mathrm{P}<0.05$ were considered statistically significant. 


\section{Results}

One hundred and nineteen patients were eligible and agreed to enter the study after forty-one patients excluded. Sixty patients and fifty-nine patients were randomized into the group KFEH and group SPT groups, respectively. All of the enrolled patients only underwent TKA of one knee. Four patients in the group SPT did not receive the allocated intervention for distance inconvenient to hospital or rehabilitation cost. One patient in group KFEH was withdrawn because she had fracture for falling at 3 month postoperative. Three patients in group KFEH and two patients in group SPT were withdrawn from analyzing for getting other system disease postoperatively, which required inpatient skilled nursing. One patient in group KFEH and two patients in group SPT lost to follow-up at 3 or 6 months postoperative, who did not wish to continue being evaluated. Finally 55 patients in group KFEH and 51 patients in group SPT were taken into analysis. (Fig. 1)

The mean age of the patients was $66.38 \pm 8.35$ years in the group KFEH and $67.27 \pm 6.87$ years in the group SPT. There were 11 men and 44 women in group KFEH, and group SPT included 8 men and 43 women. Other patients' characters including BMI, gender, diagnosis etc. are given in Table 1. There was no significant difference between two groups about the patients' characters.

Table 1

Mean \pm SD of patient characteristics for group KFEH and group SPT

\begin{tabular}{|lll|}
\hline Items & group KFEH $(\mathbf{n}=\mathbf{5 5})$ & group SPT $(\mathbf{n = 5 1 )}$ \\
\hline Age, $y$ & $68.4 \pm 8.4$ & $67.3 \pm 6.9$ \\
\hline Sex, $\mathrm{n}$ & & \\
Female & 44 & 43 \\
\hline Male & 11 & 8 \\
\hline Side, $\mathrm{n}$ & & \\
\hline Right & 38 & 36 \\
\hline Left & 17 & 15 \\
\hline BMl & $21.2 \pm 1.5$ & $21.5 \pm 1.6$ \\
\hline Weight, kg & $56.8 \pm 5.9$ & $57.0 \pm 5.7$ \\
\hline Height, cm & $163.8 \pm 5.2$ & $162.8 \pm 4.9$ \\
\hline Hospital stay, day & $18.27 \pm 3.76$ & $17.43 \pm 3.73$ \\
\hline
\end{tabular}

The preoperative (Table 2) and postoperative (including 1 week, 1 month, 3 month, 6 month and 1 year) data were assessed for noninferiority on each of the outcomes. Table 3 shows the clinical outcomes at different periods during 12 months follow-up. Pain and functional improvements were observed in 
postoperative assessments in both groups during 12 months follow up. But, there was no statistic difference about VAS between two groups at any time assessment. Overall ROM and functional scores, such as KSS knee and function scores and WOMAC scores, were slightly better in low stool assisted home exercise program group at early follow-up. But there were no statistical difference about these clinical outcomes between group KFEH and group SPT during 12 months follow-up except the ROM ranges at 1 month after surgery $(P<0.01$, Table 3$)$. The changes between preoperative and postoperative outcomes of each patient also showed group KFEH had better improvements of ROM at early follow up (Fig. 2).

Table 2

Mean \pm SD of preoperative data

\begin{tabular}{|llll|}
\hline Outcome & group KFEH & group SPT & $P$ \\
\hline KSS knee score, points & $46.7 \pm 12.0$ & $44.2 \pm 14.6$ & 0.61 \\
\hline KSS function score, points & $42.0 \pm 11.6$ & $44.0 \pm 12.0$ & 0.79 \\
\hline ROM, deg & $100.0 \pm 10.1$ & $101.0 \pm 10.2$ & 0.58 \\
\hline VAS, points & $4.3 \pm 2.2$ & $4.4 \pm 2.2$ & 0.83 \\
\hline WOMAC, points & $52.7 \pm 9.6$ & $51.3 \pm 11.4$ & 0.18 \\
\hline $\begin{array}{l}\text { Abbreviations: KSS, Knee Society score; ROM, Range of Motion; VAS, Visual Analogue Scale; WOMAC, } \\
\text { Western Ontario and McMaster Universities Osteoarthritis Index. }\end{array}$ \\
\hline
\end{tabular}


Table 3

Postoperative outcome by treatment group (Mean \pm SD)

\section{Outcome}

KSS knee score, points

Wk 1

Mo 1

Mo 3

Mo 6

$\mathrm{Yr} 1$

KSS function score, points

Wk 1

Mo 1

Mo 3

Mo 6

Yr 1

ROM, deg

Wk 1

Mo 1

Mo 3

Mo 6

Yr 1

VAS, points

Wk 1

Mo 1

Mo 3

Mo 6

Yr 1

WOMAC, points

Abbreviations: SD, standard deviation; deg, degrees; Wk, week; Mo, month; $Y r$, year; KSS, Knee Society score; ROM, Range of Motion; VAS, Visual Analogue Scale; WOMAC, Western Ontario and McMaster Universities Osteoarthritis Index. group KFEH

$51.7 \pm 14.9$

$75.3 \pm 9.6$

$82.3 \pm 7.3$

$88.3 \pm 7.2$

$88.8 \pm 7.8$

$38.2 \pm 15.0$

$61.6 \pm 11.3$

$76.8 \pm 13.1$

$86.7 \pm 11.1$

$90.2 \pm 10.1$

$86.2 \pm 12.9$

$88.2 \pm 10.3$

0.40

$99.4 \pm 8.5$

$107.9 \pm 10.5$

$114.0 \pm 10.4$

$115.3 \pm 8.2$

$5.2 \pm 1.7$

$5.5 \pm 1.2$

0.42

$2.6 \pm 1.1$

$2.7 \pm 1.3$

0.49

$1.7 \pm 1.0$

$1.6 \pm 1.1$

0.90

$1.1 \pm 1.0$

$1.0 \pm 0.9$

0.61

$0.9 \pm 0.8$

$0.8 \pm 0.8$

0.75 


\begin{tabular}{|llll|}
\hline Outcome & group KFEH & group SPT & $P$ \\
\hline Wk 1 & $57.5 \pm 14.4$ & $58.7 \pm 11.5$ & 0.63 \\
\hline Mo 1 & $34.2 \pm 10.6$ & $38.0 \pm 13.8$ & 0.12 \\
\hline Mo 3 & $25.9 \pm 11.7$ & $16.7 \pm 12.4$ & 0.76 \\
\hline Mo 6 & $15.2 \pm 10.0$ & $14.4 \pm 9.6$ & 0.67 \\
\hline Yr 1 & $9.2 \pm 9.1$ & $9.9 \pm 8.2$ & 0.68 \\
\hline $\begin{array}{l}\text { Abbreviations: SD, standard deviation; deg, degrees; Wk, week; Mo, month; Yr, year; KSS, Knee Society } \\
\text { score; ROM, Range of Motion; VAS, Visual Analogue Scale; WOMAC, Western Ontario and McMaster } \\
\text { Universities Osteoarthritis Index. }\end{array}$ & & \\
\hline
\end{tabular}

The average total costs analysis of first 2 months for supervised physiotherapy and knee flexion enhanced standardized home exercise program was performed. The approximate total costs were 1805 RMB in group SPT and 1023 RMB in group KFEH. Patients in group KFEH could save about 800 RMB after 2 months rehabilitation (details in Table 4). 
Table 4

Cost analysis of group KFEH and group SPT (monetary RMB)

\begin{tabular}{|lll|}
\hline Applications & group KFEH & group SPT \\
\hline Assessment Price & $140(20 \times 7)$ & $140(20 \times 7)$ \\
\hline Preoperative assessment & 20 & 20 \\
\hline Outpatients clinic assessment & 20 & 20 \\
\hline Assessment at 2 week & 20 & 20 \\
\hline Assessment at 1 month & 20 & 20 \\
\hline Assessment at 3 months & 20 & 20 \\
\hline Assessment at 6 months & 20 & 20 \\
\hline Assessment at 12 months & 20 & 20 \\
\hline Physiotherapy items & 105 & $1505(100 \times 14+105)$ \\
\hline Weekly postoperative guide & $105(15 \times 7)$ & $105(15 \times 7)$ \\
\hline Warm heat application & 0 & 10 \\
\hline ROM and strength exercises & 0 & 45 \\
\hline TENS & 0 & 45 \\
\hline Exercise Equipments & 730 & 0 \\
\hline Low stool & 30 & 0 \\
\hline Stationary bicycle & 700 & 0 \\
\hline Transportation fee(round trip) & $48(8 \times 6)$ & $160(8 \times 20)$ \\
\hline Total cost & 1023 & 1805 \\
\hline
\end{tabular}

As to the complication, no DVT, infection or tendon tears during exercise was observed in either group. There was only one patient in group KFEH had fracture after falling out at 3 month followed in hospital care, who was excluded from data analysis at last.

\section{Discussion}

After 12 months follow-up assessment, our study observed good outcomes at first month in the patients who took knee flexion enhanced home exercise program. Group KFEH had better ROM range at 1 month than group SPT $(P<0.01)$. No inferiority was shown about the value of WOMAC, KSS and VAS between two groups as well as complication incidence rate. Not surprisingly, present study showed that home program would lower patients' times and economic burden. 
Total knee arthroplasty is an effective treatment for end stage arthritis, relieving millions of patients suffering arthritis and improving the quality of life [20]. With the process of ageing society, the number of patients undertook TKA surgery has been increased throughout the world in recent years [21, 22]. Helping patients return to their pre-disease conditions and improve quality of life is the meaning of this surgery. Besides excellent surgeon's operation, postoperative rehabilitation is considered to have much affection about patients' knees function and satisfaction towards surgeries [23, 24]. Faced with high cost of physiotherapy, an effective home program protocol needs to expand in China. By chance, we observed that a crowed of patients who had habitat of sitting small low stools gained fast rehabilitation and better satisfaction after TKA surgery. We hypothesize that sitting low stool could improve ROM especially knee flexion and help patients get faster and better rehabilitation after TKA surgery. Just as some studies demonstrated that the increasing ROM is important for patients' functional outcome and satisfaction after TKA $[25,26]$.

Knee ROM is an objective variable to evaluate final flexion after total knee arthroplasty. With a postoperative ROM between 100 degrees and 120 degrees, most activities of daily life can be performed comfortably $[27,28]$. The knee ROM usually decreased after TKA surgery comparing with preoperative conditions because of surgery region discomfort, such as incision pain, knee swelling and prosthesis unfamiliar. But most of patients can have knee flexion more than 90 degrees 3 or 4 days postoperative after in hospital rehabilitation according to our experience. One of challenge home rehabilitation has to face with is unsatisfied knee flexion rehabilitation out of hospital due to patients unclear recover aim and poorly exercised. The advantage of KFEH program is to establish a proper target and self-ware rehabilitation test when we ask patients in group KFEH could do flexion practice while sitting a low stool. In our study, ROM showed increasing trend after surgery in both groups. There is obvious difference at the first month, which showed low stool assisted home exercise program group had larger range of knee joint motion ( $99.4 \pm 8.5$ degrees and $94.2 \pm 9.8$ degrees respectively, $P<0.01)$. The change of ROM between postoperative and preoperative also had similar results (Fig. 2), as well as the absolute values of the KSS pain and function scores and the WOMAC scores, which reflect that patient satisfaction was somewhat better in group KFEH. All of these outcomes results suggest that low stool might improve knee joint range of motion after TKA.

Knee Society Score (KSS) is a clinical rating system published in 1989 to measure the knee in patients having TKA [29]. The Western Ontario and McMaster Universities Osteoarthritis Index (WOMAC) is developed to evaluate the pain, stiffness and functional limitation condition of patients who suffered osteoarthritis by Bellamy in 1982 [30]. Both of KSS and WOMAC could evaluate knee function and present patients' satisfaction towards postoperative rehabilitation. In our study, there were no significant differences between group KFEH and group SPT about KSS or WOMAC results, though absolute values of the KSS and the WOMAC scores were better in group KFEH during beginning 3 months after surgery. While several factors contributing to knee function or satisfaction and outcomes vary from patient to patient. Some studies demonstrated that patient perceptions of function could differ from actual function, and patient factors including obesity, motivation, fatigue etc. could also affect results [11, 26 , 
31]. This may explain ROM difference, an individual factor change, is unlikely to completely influence KSS or WOMAC results.

Physiotherapy cost and inconvenience is a source of concern for patients in group SPT after TKA. Standard supervised physiotherapy treatment usually includes warm heat application, ROM exercises, strength recovery and other applications to avoid postoperative conditions such as loss of motion of the joint, muscle atrophy, tissue edema, functional limitations etc [32, 33]. It is recommended that patients should receive physiotherapy training two or three times weekly to achieving these goals [34]. An American study demonstrated that medicare reimbursements for physical therapy would over 1000 dollar for 12 sessions, and home rehabilitation can much lower the economic burden on patients [11]. In our study, home exercise program involves several actions to rehabilitate ROM of joint, muscle strength, and gait balance. Small low stools would help patients to enhance the flexion of knee joint during exercise. The total cost of each groups mainly contain assessments and training applications. The approximate total costs of 2 months rehabilitation were 1805 RMB in physiotherapy group and 1023 RMB in home exercise group after roughly analysis (Table 4). In consideration of the resident income of China in 2016 was 23821 RMB [35], home exercise program would lower the economic burden of the patients taking undervalue China medical staffs' work into account.

Besides function recovery, we also concern whether the procedure of enhancing knee flexion by sitting low stool would cause ligaments injury during rehabilitation. After 12 month follow-up, there was no records reflects patients had ligaments tears or severe pain around the knee joint. DVT, infection or other complications were not occurred as well. Above all, the safety of low stool assisted home exercise program is considered to be noninferior compared with supervised physiotherapy.

This study had some limitations. First, assessor physiotherapist was not blind. Second, the patient compliance of group KFEH is good because of regular follow-up and scoring, but it is not guaranteed that we could get same results from fair or worse compliance patients, such as the ones refused to take part in the study or ones fed up with long-term follow-up. Third, physical therapists differed for each patient, as well as the details of therapy protocol and frequency, and this would result individual difference inevitable. Last, the number of samples is not enough to evaluate some outcomes difference between two groups, and studies with more participants are necessary.

\section{Conclusion}

Our finding suggests that the knee flexion enhanced standardized home exercise program had better ROM at early discharge. The VAS, KSS score, WOMAC score, and complication rates during 12 months follow-up were noninferior compared with supervised physiotherapy. Above all, we verified the efficiency and economic of using KFEH for TKA rehabilitation management.

\section{Abbreviations}


KSS knee society score

OA osteoarthritis

QF $\quad$ quadriceps femoris

ROM range of motion

TENS transcutaneous electrical nerve stimulation

TKA total knee arthroplasty

VAS visual analogue scale

WOMAC the Western Ontario and McMaster Universities Osteoarthritis Index

\section{Declarations}

\section{Compliance with Ethical Standards}

Conflict of Interest: The authors declare that they have no conflict of interest.

Funding: This work was supported by the National Natural Science Foundation of China (NSFC), grant number (81572632).

Ethical approval: This article was approved by the Institutional Medical Ethics Committee of our hospital.

\section{Ethics approval and consent to participate[}

The experimental protocol was established, according to the ethical guidelines of the Helsinki Declaration and was approved by the Human Ethics Committee of Shanghai Tenth People's Hospital. Written informed consent was obtained from individual or guardian participants.

\section{Consent for publication $\llbracket$}

Not applicable.

\section{Availability of data and material:}

All data generated or analysed during this study are included in this published article.

\section{Competing interests $\rrbracket$}

The authors declare that they have no competing interests 
Authors' contributions】

T.Xu and G.Li conceived and designed the study. L.Fan,D.Yang and K.Liu collected and processed the data. T.Xu wrote the paper. T.Xu,Q.Gao, D.Yang and K.Liu reviewed and edited the manuscript. All authors read and approved the final manuscript.

\section{Acknowledgements \}

We really appreciate the colleagues from our department for helping to solve related issues.

\section{References}

1. Losina E, Paltiel AD, Weinstein AM, Yelin E, Hunter DJ, Chen SP, Klara K, Suter LG, Solomon DH, Burbine SA, Walensky RP, Katz JN. Lifetime medical costs of knee osteoarthritis management in the United States: impact of extending indications for total knee arthroplasty. Arthritis Care Res. 2015;67:203-15. DOI 10.1002/acr.22412.

2. Rampersaud YR, Tso P, Walker KR, Lewis SJ, Davey JR, Mahomed NN, Coyte PC. Comparative outcomes and cost-utility following surgical treatment of focal lumbar spinal stenosis compared with osteoarthritis of the hip or knee: part 2-estimated lifetime incremental cost-utility ratios. The spine journal: official journal of the North American Spine Society. 2014;14:244-54. DOI 10.1016/j.spinee.2013.11.011.

3. Pozzi F, Snyder-Mackler L, Zeni J. Physical exercise after knee arthroplasty: a systematic review of controlled trials. Eur J Phys Rehabil Med. 2013;49:877-92.

4. Smith WA, Zucker-Levin A, Mihalko WM, Williams M, Loftin M, Gurney JG. Physical Function and Physical Activity in Obese Adults After Total Knee Arthroplasty. The Orthopedic clinics of North America. 2017;48:117-25. DOI 10.1016/j.ocl.2016.12.002.

5. Paxton RJ, Melanson EL, Stevens-Lapsley JE, Christiansen CL. Physical activity after total knee arthroplasty: A critical review. World journal of orthopedics. 2015;6:614-22. DOI 10.5312/wjo.v6.i8.614.

6. Ferreira GE, Robinson CC, Wiebusch M, Viero CC, da Rosa LH, Silva MF. The effect of exercise therapy on knee adduction moment in individuals with knee osteoarthritis: A systematic review. Clin Biomech (Bristol Avon). 2015;30:521-7. DOI 10.1016/j.clinbiomech.2015.03.028.

7. Hussain SM, Neilly DW, Baliga S, Patil S, Meek R. Knee osteoarthritis: a review of management options. Scot Med J. 2016;61:7-16. DOI 10.1177/0036933015619588.

8. Moyer R, lkert K, Long K, Marsh J. The Value of Preoperative Exercise and Education for Patients Undergoing Total Hip and Knee Arthroplasty: A Systematic Review and Meta-Analysis. JBJS reviews. 2017;5:e2. DOI 10.2106/JBJS.RVW.17.00015.

9. Rutherford RW, Jennings JM, Dennis DA. Enhancing Recovery After Total Knee Arthroplasty. The Orthopedic clinics of North America. 2017;48:391-400. DOI 10.1016/j.ocl.2017.05.002. 
10. Florez-Garcia M, Garcia-Perez F, Curbelo R, Perez-Porta I, Nishishinya B, Rosario Lozano MP, Carmona L. (2016) Efficacy and safety of home-based exercises versus individualized supervised outpatient physical therapy programs after total knee arthroplasty: a systematic review and meta-analysis. Knee surgery, sports traumatology, arthroscopy: official journal of the ESSKA. DOI 10.1007/s00167016-4231-x.

11. Levine M, McElroy K, Stakich V, Cicco J. Comparing conventional physical therapy rehabilitation with neuromuscular electrical stimulation after TKA. Orthopedics. 2013;36:e319-24. DOI 10.3928/01477447-20130222-20.

12. Buker N, Akkaya S, Akkaya N, Gokalp O, Kavlak E, Ok N, Kiter AE, Kitis A. Comparison of effects of supervised physiotherapy and a standardized home program on functional status in patients with total knee arthroplasty: a prospective study. J Phys Ther Sci. 2014;26:1531-6. DOI 10.1589/jpts.26.1531.

13. Unver B, Bakirhan S, Karatosun V. Does a weight-training exercise programme given to patients four or more years after total knee arthroplasty improve mobility: A randomized controlled trial. Arch Gerontol Geriatr. 2016;64:45-50. DOI 10.1016/j.archger.2016.01.003.

14. Matassi F, Duerinckx J, Vandenneucker H, Bellemans J. Range of motion after total knee arthroplasty: the effect of a preoperative home exercise program. Knee surgery, sports traumatology, arthroscopy:. official journal of the ESSKA. 2014;22:703-9. DOI 10.1007/s00167-012-2349-z.

15. (2005) Physiotherapy rehabilitation after total knee or hip replacement: an evidence-based analysis. Ontario health technology assessment series 5:1-91.

16. Robbins CE, Bono JV, Ward DM, Barry MT, Doren J, McNinch A. Effect of preoperative exercise on postoperative mobility in obese total joint replacement patients. Orthopedics. 2010;33:666. DOI 10.3928/01477447-20100722-09.

17. Scott DF. (2011) Knee Joint Replacement Surgery Post-Operative Exercise Program. In.

18. Monticone M, Ferrante S, Rocca B, Salvaderi S, Fiorentini R, Restelli M, Foti C. Home-based functional exercises aimed at managing kinesiophobia contribute to improving disability and quality of life of patients undergoing total knee arthroplasty: a randomized controlled trial. Arch Phys Med Rehabil. 2013;94:231-9. DOI 10.1016/j.apmr.2012.10.003.

19. Ge DJ, Qi B, Tang G, Li JY. Intraoperative Dexmedetomidine Promotes Postoperative Analgesia and Recovery in Patients after Abdominal Hysterectomy: a Double-Blind, Randomized Clinical Trial. Scientific reports. 2016;6:21514. DOI 10.1038/srep21514.

20. George J, Navale SM, Schiltz NK, Siccha M, Klika AK, Higuera CA. (2016) Racial Disparities in Aboveknee Amputations After TKA: A National Database Study. Clinical orthopaedics and related research. DOI 10.1007/s11999-016-5195-3.

21. Skou ST, Roos EM, Laursen MB, Rathleff MS, Arendt-Nielsen L, Simonsen O, Rasmussen S. A Randomized, Controlled Trial of Total Knee Replacement. N Engl J Med. 2015;373:1597-606. DOI 10.1056/NEJMoa1505467. 
22. Akgun I, Ogut T, Kesmezacar H, Yucel I. [Total knee arthroplasty: a 4.5-year follow-up]. Acta Orthop Traumatol Turc. 2002;36:93-9.

23. Quack V, Ippendorf AV, Betsch M, Schenker H, Nebelung S, Rath B, Tingart M, Luring C.

[Multidisciplinary Rehabilitation and Fast-track Rehabilitation after Knee Replacement: Faster, Better, Cheaper? A Survey and Systematic Review of Literature]. Die Rehabilit. 2015;54:245-51. DOI 10.1055/s-0035-1555887.

24. Bade MJ, Stevens-Lapsley JE. Restoration of physical function in patients following total knee arthroplasty: an update on rehabilitation practices. Curr Opin Rheumatol. 2012;24:208-14. DOI 10.1097/BOR.0b013e32834ff26d.

25. Matsuda S, Kawahara S, Okazaki K, Tashiro Y, Iwamoto Y. Postoperative alignment and ROM affect patient satisfaction after TKA. Clin Orthop Relat Res. 2013;471:127-33. DOI 10.1007/s11999-0122533-y.

26. Ha CW, Park YB, Song YS, Kim JH, Park YG. Increased Range of Motion Is Important for Functional Outcome and Satisfaction After Total Knee Arthroplasty in Asian Patients. J Arthroplasty. 2016;31:1199-203. DOI 10.1016/j.arth.2015.12.018.

27. Laubenthal KN, Smidt GL, Kettelkamp DB. A quantitative analysis of knee motion during activities of daily living. Physical therapy. 1972;52:34-43.

28. Crijns Yh Fau -

Lenssen AF, Crijns Yh Fau - Waltje EMH. Waltje Em Fau - Roox GM, Roox Gm Fau - van Steyn MJA, van Steyn Mj Fau - Geesink RJT, Geesink Rj Fau - van den Brandt PA, van den Brandt Pa Fau - de Bie RA, de Bie RA Effectiveness of prolonged use of continuous passive motion (CPM) as an adjunct to physiotherapy following total knee arthroplasty: design of a randomised controlled trial [ISRCTN85759656].

29. Insall JN, Dorr LD, Scott RD, Scott WN. (1989) Rationale of the Knee Society clinical rating system. Clinical orthopaedics and related research:13-14.

30. Bellamy N, Buchanan WW, Goldsmith $\mathrm{CH}$, Campbell J, Stitt LW. Validation study of WOMAC: a health status instrument for measuring clinically important patient relevant outcomes to antirheumatic drug therapy in patients with osteoarthritis of the hip or knee. J Rhuematol. 1988;15:1833-40.

31. Piva SR, Fitzgerald GK, Irrgang JJ, Bouzubar F, Starz TW. Get up and go test in patients with knee osteoarthritis. Arch Phys Med Rehabil. 2004;85:284-9.

32. Coppola SM, Collins SM. Is physical therapy more beneficial than unsupervised home exercise in treatment of post surgical knee disorders? A systematic review. Knee. 2009;16:171-5. DOI 10.1016/j.knee.2008.09.001.

33. Park D, Kim J, Lee H. Effectiveness of Modified Quadriceps Femoris Muscle Setting Exercise for the Elderly in Early Rehabilitation after Total Knee Arthroplasty. J Phys Ther Sci. 2012;24:27-30.

34. Maruyama T, Sawada Y, Kubo S, Kinoshita K, Kurosaka M, Shimada T. Postoperative Changes in Knee Joint Function of Total Knee Arthroplasty Patients. J Phys Ther Sci. 2011;23:719-24. 
35. Bureau NS (2016) Statistical Bulletin of 2016. In. < http://www.stats.gov.cn/tjsj/sjjd/201702/t20170228_1467357.html>

\section{Figures}

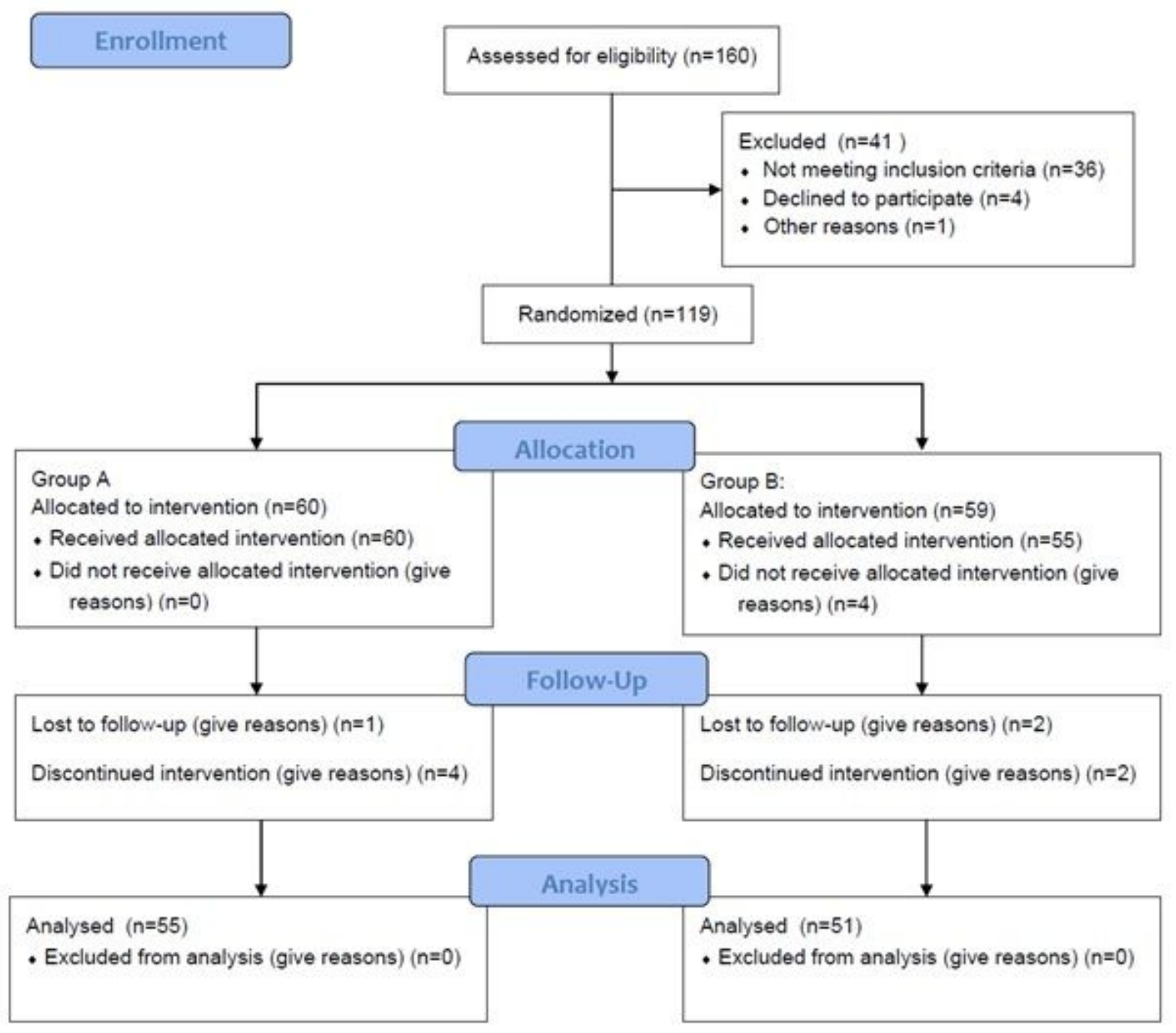

\section{Figure 1}

Flow diagram of this study 

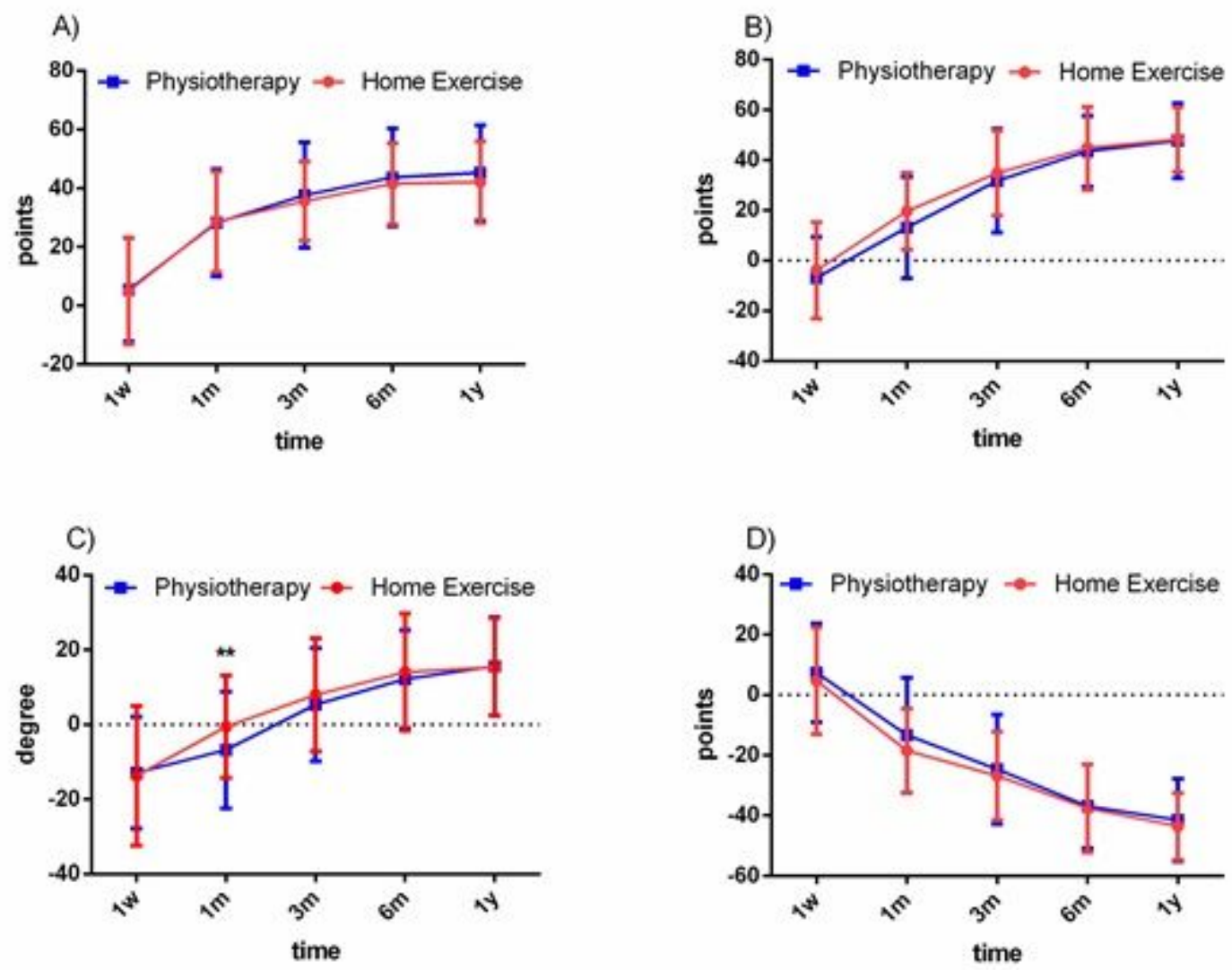

Figure 2

Differences of clinical outcomes between postoperative and reoperative A) KSS knee score; B) KSS function score; C) ROM; D) WOMAC score

\section{Supplementary Files}

This is a list of supplementary files associated with this preprint. Click to download.

- CONSORT2010Checklist.pdf

- Appendixl.pdf 